CITATION

Rhee, L., Bayer, J. B., \& Hedstrom, A. (2020). Experience sampling method. In J. Van den Bulck (Ed.), The International Encyclopedia of Media Psychology. John Wiley \& Sons.

\title{
Experience Sampling Method
}

\author{
Lisa Rhee, Joseph B. Bayer, and Alex Hedstrom
}

The Ohio State University, Columbus, USA

\begin{abstract}
The use of experience sampling methodology (ESM), also known as ecological momentary assessment (EMA), is firmly established in the social sciences. The family of ESM methods employs in vivo self-reports, providing opportunities for social scientists to study human behavior with high ecological validity. More recently, researchers across disciplines have begun to conduct studies that directly combine ESM with mobile sensing, thereby blending the benefits of subjective self-reports with more objective traces of human behavior. This combination of ESM and mobile sensing offers new opportunities, such as predetermined digital events that initiate ESM survey notifications based on contextual information. Altogether, ESM is evolving in ways that offer new opportunities and challenges for researchers.
\end{abstract}

\section{Keywords}

ambulatory assessment; context; digital trace; ecologicalmomentaryassessment; EMA; ESM; event; triggers

Experience sampling method (ESM) is used by researchers across a wide range of disciplines to gather insights into human behavior in daily life. The method employs in situ self-reports for which participants are instructed to answer a short questionnaire upon receiving a signal. As ESM self-reports are administered in a naturalistic setting, as opposed to an artificial laboratory environment, they provide a more immediate, and potentially accurate, representation of lived behavior. As such, this method is often used to observe fleeting and subjective experiences (e.g., mood, stress) that are best assessed in the moment. In the process, researchers can leverage the benefits of more robust statistical techniques and conduct more targeted analyses of real-world behaviors which occur less frequently. ESM exhibits methodological similarities to traditional diary studies, wherein participants are asked to log key information about their behavior over time. Likewise, ESM is comparable to the day reconstruction method (DRM), in which participants 
report on recent experiences by separating their past day into major activities, such as meals and commutes (Diener \& Tay, 2014). Unlike these methods, however, ESM aims to provide a more granular approach by minimizing the participants' use of long-term memory to reproduce past experiences. In sum, ESM represents one of the predominant approaches for studying phenomena with ecological validity in mind (van Berkel, Ferreira, \& Kostakos, 2017).

The quintessential element of ESM is the prompting of participants to complete a self-report questionnaire in daily life. From the early days of ESM, researchers have adopted different approaches to prompt participants (Scollon, Kim-Prieto, \& Scollon, 2003). Whereas some studies have triggered participants to initiate questionnaires based on prescheduled intervals or random times of day, others have done so based on predefined events. In the former, researchers attempt to gather samples at a representative schedule of times, while avoiding expectancy effects that rise from having a prior knowledge of the sampling period (Scollon et al., 2003). Alternatively, if anticipatory effects are deemed too burdensome or research questions are more context specific, event-contingent triggers can be used to target key situations (e.g., after every social interaction; Moskowitz \& Côté, 1998), with early studies typically relying on participants recognizing said events. In practice, many researchers now apply various approaches in their contingency configurations to match study needs.

\section{Mobile Devices and Sensors}

Early ESM studies used electronic radio pagers to prompt participants who would then complete a paper-based self-report (e.g., Moskowitz \& Côté, 1998). By the 1980s, studies began to use computerized methods of data collection, the advent of what scholars increasingly refer to as "mobile" experience sampling (Hedstrom \& Irwin, 2017). The use of computerized methods such as personal data assistants (PDAs) allowed researchers to have better control over the timing of triggers and eliminated the costs of coding and entering data (Hektner, Schmidt, \& Csikszentmihalyi, 2007). However, there were drawbacks to these early mobile devices, such as limited battery life, unstable data storage, and high device costs. Furthermore, participants had to carry around the devices in their daily lives and go through training procedures to learn how to use them. The shortcomings of the earlier ESM tools are partially overcome with the rise of mobile devices owned by individuals, which has given researchers a more convenient vehicle for triggering and collecting surveys.

As such, personal devices have been leveraged to efficiently distribute ESM questionnaires by message, email, or app. Beyond sending in vivo survey notifications, contemporary devices can also be used to collect data from the associated digital records or traces vis-à-vis embedded sensors. Such "sensing" allows researchers to unobtrusively gather objective information about a person, their device, and/or their environment (Miluzzo et al., 2008). Researchers can now gather a diverse collection of real-time data from the commonly supplied microphone, camera, accelerometer, global-positioning system (GPS), Bluetooth radio, WiFi scans, ambient light sensor, gyroscope, and thermometer (Lane et al., 2010) as well as device activities and user heart rate (Pejovic, Lathia, Mascolo, \& Musolesi, 2016). From these digital traces, researchers are able to study in situ social interactions, media behaviors, mobility patterns, and spatial environments (e.g., Harari et al., 2017). 


\section{Digital Triggers and Annotations}

The aforementioned sensors also allow for a new class of automated event-based triggers. Researchers have long sought to develop context-aware systems that extract, interpret, and use contextual information. Through such systems, researchers can set context triggers: predetermined events that initiate ESM notifications based on contextual information (Pejovic et al., 2016; van Berkel et al., 2017). Context triggers allow social science researchers to collect in vivo self-report data in the context defined by theoretical questions, and without requiring participants to be constantly vigilant. Moreover, researchers can gather a wide-ranging collection of specific behaviors, applications, or activities that covary with the preselected contexts (e.g., Bayer, Ellison, Schoenebeck, Brady, \& Falk, 2018; Chang, Paruthi, Wu, Lin, \& Newman, 2017).

In addition to unobtrusive triggering around "digital events," sensing tools offer ways to improve the quality of self-report data by providing visual cues or visualizations relevant to past behaviors. Such methods are reminiscent of the event history calendar (or EHC) surveying approach, which has been demonstrated to improve data quality in terms of completeness and consistency (Sayles, Belli, \& Serrano, 2010). This calendar-based technique facilitates recall accuracy through graphical presentation of timelines that closely reflect the structure of participants' autobiographical memory (Belli, 1998). In a similar manner, through digital logs, researchers can show participants their behavioral traces as reference material to help them recollect and "annotate" their past behavior (Chang et al., 2017; Intille, Kukla, \& Ma, 2002). In sum, current devices allow unprecedented opportunities for social science researchers to collect new forms of subjective and objective data that augment the original goals ofESM.

\section{Opportunities and Challenges}

Although contemporary approaches have reduced major drawbacks of traditional ESM, they still present novel challenges. For any sensor-triggered study, researchers must decide when and under what conditions a survey notification should be delivered. This is critical not only in terms of improving user interaction and compliance, but also due to the potential of bias in the resultant data (Pejovic et al., 2016). Past research has indicated that design choices influence the inferences that can be made from such studies, especially when using single-sensor sampling strategies (Lathia \& Rachuri, 2013). As a single-sensor sampling strategy can produce skewed results, it was suggested that sampling could be improved by using multiple sensors to determine a trigger (Lathia \& Rachuri, 2013). Additionally, researchers struggle with how to capture the daily experiences of participants without interfering with their lifestyles, as well as how to sample moments across highly variable personal schedules (Pejovic et al., 2016).

Finally, the use of mobile computing devices for ESM studies introduces technical challenges to the researchers, including "inter-device communication across devices and software systems, strict battery optimisation techniques, and different input techniques between devices" (van Berkel et al., 2017, p. 28). For example, programming mobile applications and managing mobile sensing systems require advanced technical setup and analytic skills (Harari, Müller, \& Gosling, 2018; Pejovic et al., 2016). Additionally, mobile sensing studies face ethical issues in relation to the study design and execution, such as maintaining researcher transparency and safeguarding 
participant data (Harari et al., 2018). Despite these challenges, ESM continues to be an integral tool for social scientists. Furthermore, modern approaches have transformed traditional ESM through integration with mobile sensing, unveiling increasingly nuanced ways to assess in vivo human behavior. At their core, ESM studies offer an unmatched lens into everyday situations, behaviors, and cognitions, and the ensuing views become sharper as the method evolves.

\section{References}

Bayer, J., Ellison, N., Schoenebeck, S., Brady, E., \& Falk, E. B. (2018). Facebook in context(s): Measuring emotional responses across time and space. New Media and Society, 20(3), 10471067. doi:10.1177/1461444816681522

Belli, R. F. (1998). The structure of autobiographical memory and the event history calendar: Potential improvements in the quality of retrospective reports in surveys. Memory, 6(4), 383406. doi:10.1080/741942610

Chang, Y. J., Paruthi, G., Wu, H. Y., Lin, H. Y., \& Newman, M. W. (2017). An investigation of using mobile and situated crowdsourcing to collect annotated travel activity data in real-word settings. International Journal of Human Computer Studies, 102, 81-102. doi:10.1016/j.ijhcs.2016.11.001

Diener, E., \& Tay, L. (2014). Review of the day reconstruction method (DRM). Social Indicators Research, 116(1), 255-267. doi:10.1007/s11205-013-0279-x

Harari, G. M., Gosling, S. D., Wang, R., Chen, F., Chen, Z., \& Campbell, A. T. (2017). Patterns of behavior change in students over an academic term: A preliminary study of activity and sociability behaviors using smartphone sensing methods. Computers in Human Behavior, 67, 129-138. doi:10.1016/j.chb.2016.10.027

Harari, G. M., Müller, S. R., \& Gosling, S. D. (2018). Naturalistic assessment of situations using mobile sensing methods. In J. F. Rauthmann, R. A. Sherman, \& D. C. Funder (Eds.), The Oxford handbook of psychological situations (pp. 1-28). Oxford: Oxford University Press. doi:10.1093/oxfordhb/9780190263348.013.14

Hedstrom, A., \& Irwin, M. (2017). Mobile experience sampling method (MESM). In J. Matthes, C. S. Davis, \& R. F. Potter (Eds.), The international encyclopedia of communication research methods. New York, NY: John Wiley \& Sons. doi:10.1002/9781118901731.iecrm0157

Hektner, J. M., Schmidt, J. A., \& Csikszentmihalyi, M. (2007). Experience sampling method: Measuring the quality of everyday life. Thousand Oaks, CA: Sage.

Intille, S. S., Kukla, C., \& Ma, X. (2002). Eliciting user preferences using image-based experience sampling and reflection. In CHI 2002: Extended abstracts on human factors in computing systems (pp. 738-739). New York, NY: ACM. doi:10.1145/506443.506573

Lane, N. D., Miluzzo, E., Lu, H., Peebles, D., Choudhury, T., \& Campbell, A. T. (2010). A survey of mobile phone sensing. IEEE Communications Magazine, 48(9), 140-150. doi:10.1109/ MCOM.2010.5560598

Lathia, N., \& Rachuri, K. (2013). Contextual dissonance: Design bias in sensor-based experience sampling methods. In Proceedings of the 2013 ACM International Joint Conference on Pervasive and Ubiquitous Computing (UbiComp '13) (pp. 183-192). New York, NY: ACM. doi:10.1145/2493432.2493452 
Miluzzo, E., Lane, N. D., Fodor, K., Peterson, R., Lu, H., Musolesi, M., ... Campbell, A. T. (2008). Sensing meets mobile social networks: The design, implementation and evaluation of the CenceMe application. In Proceedings of the 6th ACM Conference on Embedded Network Sensor Systems (pp. 337-350). New York, NY: ACM. doi:10.1145/1460412.1460445

Moskowitz, D., \& Côté, S. (1998). On the dynamic covariation between interpersonal behavior and affect: Prediction from neuroticism, extraversion, and agreeableness. Journal of Personality and Social Psychology, 75(4), 1032-1046. doi:10.1037/0022-3514.75.4.1032

Pejovic, V., Lathia, N., Mascolo, C., \& Musolesi, M. (2016). Mobile-based experience sampling for behaviour research. In M. Tkalc `ic ``, B. De Carolis, M. de Gemmis, A. Odic', \& A. Košir (Eds.), Emotions and personality in personalized services (pp. 141-161). Cham: Springer. doi:10.1007/978-3-319-31413-6

Sayles, H., Belli, R. F., \& Serrano, E. (2010). Interviewer variance between event history calendar and conventional questionnaire interviews. Public Opinion Quarterly, 74(1), 140-153. doi:10.1093/poq/nfp089

Scollon, C. N., Kim-Prieto, C., \& Scollon, C. N. (2003). Experience sampling: Promises and pitfalls, strengths and weaknesses. Journal of Happiness Studies, 4(1), 5-34. doi:10.1023/ A:1023605205115

van Berkel, N., Ferreira, D., \& Kostakos, V. (2017). The experience sampling method on mobile devices. ACM Computing Surveys, 50(6), 1-40. doi:10.1145/3123988

\section{Further Readings}

Capatu, M., Regal, G., Schrammel, J., Mattheiss, E., Kramer, M., Batalas, N., \& Tscheligi, M. (2014). Capturing mobile experiences: Context- and time-triggered in-situ questionnaires on a smartphone. In A. Spink, E. L. van den Broek, L. Loijens, M. Woloszynowska-Fraser, \& L. Noldus (Eds.), Proceedings of Measuring Behavior (pp. 27-30). Wageningen: Noldus Information Technology.

Chen, Z., Chen, Y., Hu, L., Wang, S., Jiang, X., Ma, X., ... Campbell, A. T. (2014). ContextSense: Unobtrusive discovery of incremental social context using dynamic bluetooth data. In Proceedings of the 2014 ACM International Joint Conference on Pervasive and Ubiquitous Computing (UbiComp '14) (pp. 23-26). New York, NY: ACM. doi:10.1002/jcp

Consolvo, S., \& Walker, M. (2003). Using the experience sampling method to evaluate ubicomp applications. IEEE Pervasive Computing, 2(2), 24-31. doi:10.1109/MPRV.2003.1203750

Hall, J. A. (2017). The regulation of social interaction in everyday life. Journal of Social and Personal Relationships, 34(5), 699-716.doi:10.1177/0265407516654580

Harari, G. M., Gosling, S. D., Wang, R., \& Campbell, A. T. (2015). Capturing situational information with smartphones and mobile sensing methods. European Journal of Personality, 29(5), 509511. doi:10.1002/per.2032

Intille, S. S., Rondoni, J., Kukla, C., Ancona, I., \& Bao, L. (2003). A context-aware experience sampling tool. In CHI'03: Extended abstracts on human factors in computer systems (pp. 972-973). New York, NY: ACM. doi:10.1145/766098.766101

Mehrotra, A., Tsapeli, F., Hendley, R., \& Musolesi, M. (2017). MyTraces: Investigating correlation and 
causation between users' emotional states and mobile phone interaction. In Proceedings of the ACM on Interactive, Mobile, Wearable and Ubiquitous Technologies (Vol. 1, pp. 1-21). New York, NY: ACM. doi:10.1145/3130948

Lisa Rhee is a PhD student in the School of Communication at The Ohio State University and earned her BA in Communication Studies at Northwestern University. Her main research interests lie in communication technology and social interaction.

Joseph B. Bayer is an Assistant Professor at The Ohio State University and his research focuses on mobile technology, social media, and social network cognition.

Alex Hedstrom is a PhD candidate in the School of Communication at The Ohio State University, specializing in motivation, media use, and food consumption interactions. 